\title{
SIMULATION AND IMPLEMENTATION OF A WHEEL-TYPE SYSTEM ARCHITECTURE USING MODULAR ROBOTICS
}

\section{RICARDO A. CASTILLO, WILHELM ANDREWY MIÑO \& NANCY ESPERANZA OLARTE}

DAVINCI Research Group, Department of Mechatronics Engineering, Universidad Militar Nueva Granada,

\author{
Bogotá, Colombia
}

\begin{abstract}
This article presents development, simulation and implementation of a wheel-type architecture implemented using the MECABOT 4.0 modular robotic system previously designed at Universidad Militar Nueva Granada (Bogota Colombia). The main purpose of this work is to simulate (using Webots software and the C language) and implement a wheel-type configuration using eight modular robots, which will be able to offer a fast movement in a straight line from one place to another in an environment free of obstacles, and highlight the advantages that these systems bring such as: versatility in maintenance, transport and use in the various applications that require it. Functionality, stability, and speed of locomotion are evaluated through a comparative analysis between simulation and implementation results, from which a series of improvements are suggested in order to enhance speed and locomotion stability in future system versions. It was compared and analyzed positive and negative aspects of acceleration and step frequency changes for wheel-type configuration from speed as a performance indicator for the modular robotic system Mecabot 4.0.

KEYWORDS: Modular robotics, Wheel-type Architecture, Webots, MECABOT
\end{abstract}

Received: Sep 16, 2020; Accepted: Oct 06, 2020; Pbublished: Nov 11, 2020; Paper Id.: IJMPERDOCT202042

\section{INTRODUCTION}

The concept of modular robot, refers to a group of systems robotic formed by small units commonly called modules. Each of these modules has various components electronics that make this a mobile system for joints, these modules are attached to their fellows through docking interfaces (Alonso, 2006). The core of these small is module and not in the complex system that this case would be a conventional robot. The different combinations of modules are known as configurations, which allow a wide variety of designs and therefore create new modes of locomotion of the same (Arredondo, 2006).

These robots are classified by their capacity of locomotion among which are: apodo robots, whose main feature is the use of modules in series, making body movements being used in the exploration of pipes and catastrophe scenario (Bares and Wettergreen, 1999). Can also be classified as Self-propelled, which have as main characteristic that each module has an actuator which makes that each module is independentiente to the perform movements, this type can be used in military applications, inspection and surveillance (Borenstein et al, 2006).

And finally find the modular robots auto-configurable without locomotion in which modules do not have joints, these just may be prismatic or rotational (Bourkin, 2004). 


\section{PREVIOUS WORK FROM MODULAR ROBOTICS IN CONFIGURATION TYPE WHEEL}

Most of the work previously investigated was the development of typical configurations as the configuration type are snake and caterpillar. Below are some of the projects that developed about the type wheel configuration.

\section{Polybot}

In this work, each module includes two degrees of freedom driven by independent motors; each module has its own power supply, micro controller and communication infrared. The link between multiple modules is based on the union by pins and holes with mechanisms of coupling, between successful architectures is shown in Figure 1. It is in our interest, this was achieved in the latest versions of Polybot developed because the former had restrictions of movement to be the most basic modules (Lyder et al, 2008).

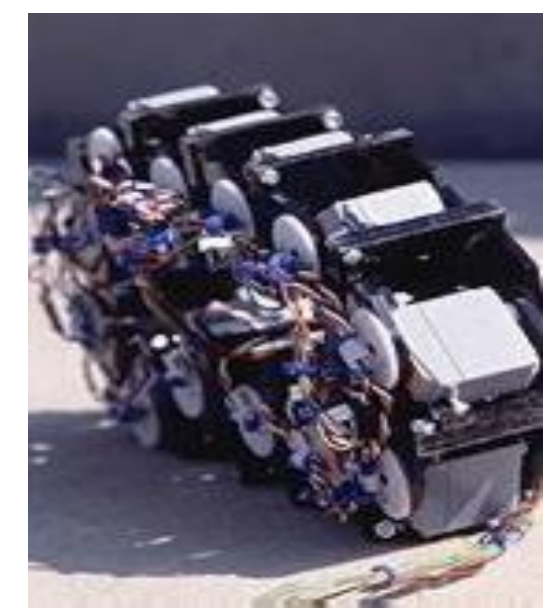

Figure 1: Polybot in Wheel Configuration (Lyder et al, 2008).

\section{Yamor}

This paper presents a modular robot based on the chain configuration; its modules have a length of $94 \mathrm{~mm}$. Each module is a self-contained system together with their own means of power, computer and communication; one of its key features is the ability to exchange information with the other modules of the configuration more Assembly a network established through a Bluetooth protocol (Ryland and Cheng, 2010). The robot yamor, in Figure 2, as well as in other hybrid robots, such as Thor (Dietsch et al, 2006), the exchange of communication not makes it possible to auto Assembly of different architectures due to the way in which the modules must join (Moeckel et al, 2007, Lyder et al, 2010).
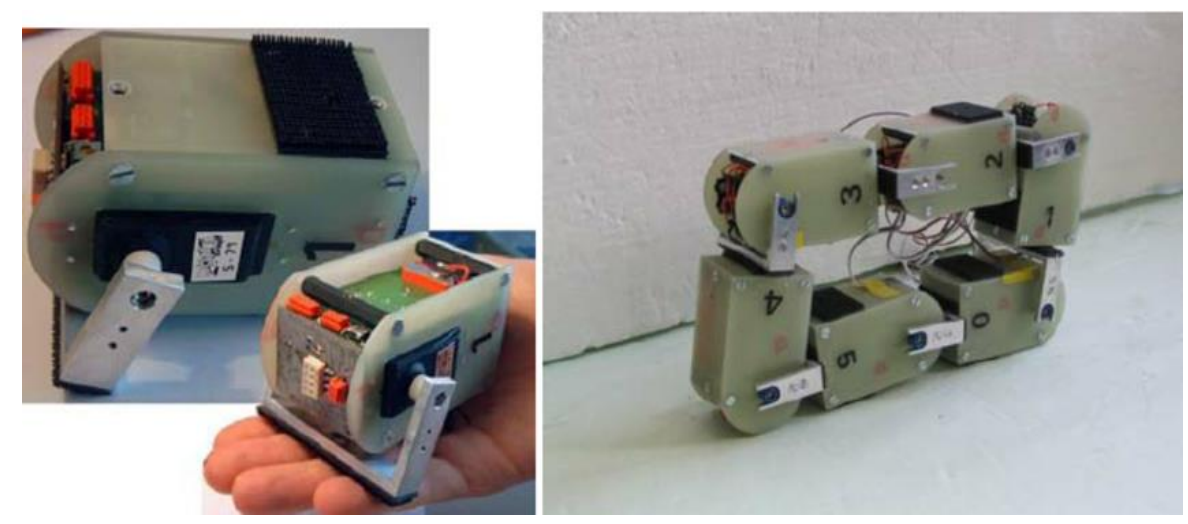

Figure 2: Yamor in Wheel Configuration (Park et al, 2008). 


\section{CKBOT (IEEE, 2005)}

This work is inspired in G1V51 model of Polybot, these modules are not auto configurable despite its complex mechanical structure, but as advantage over others is that it allows to create diverse types of configurations, modules components and some worked configurations are shown in Figure 3.

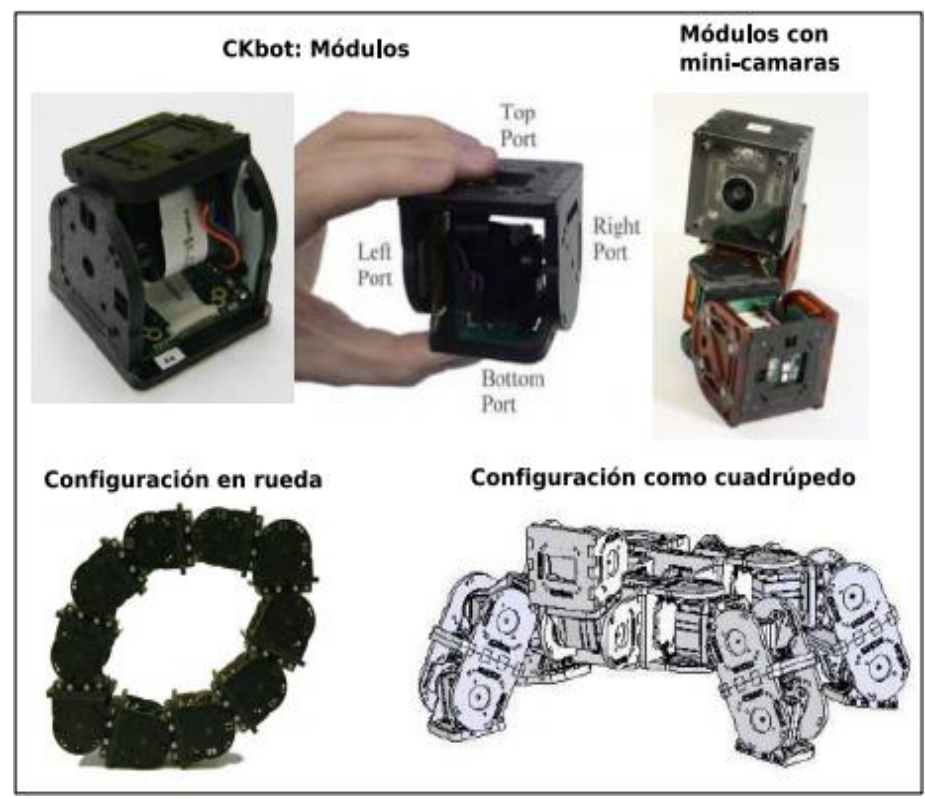

Figure 3: CKBOT in Some of Your Settings (IEEE, 2015)

\section{SIMULATION IN WEBOTS}

For this work simulations were performed using the Webots software for its tools of physical characteristics, with this simulation was developed the method of locomotion and evaluated the best way to make the movement measuring their distance displaced, time it takes to travel and speed. These tests are performed by modifying actuator parameters such as acceleration and the frequency of the movement.

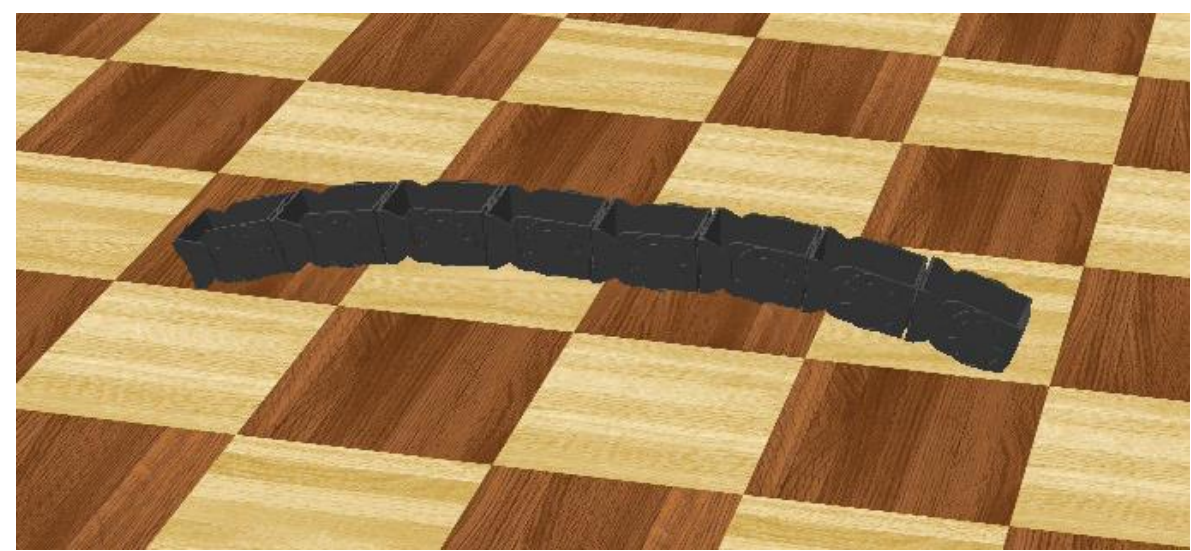

Figure 4: Architecture Type wheel in Webots

Four modules are shown in the Figure 4. implement in chain shape, this is the initial position of simulation, and modules are connected in a way pivot-center since so you can generate the necessary angles in the modules for the formation of the wheel geometry. 


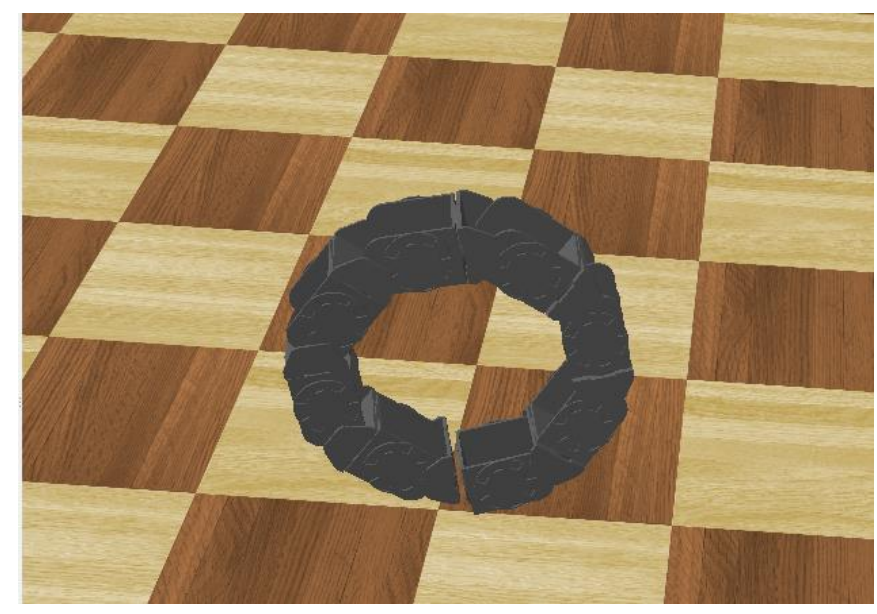

Figure 5: Simulated Rest Position

The figure 5. show the rest position of wheel configuration, where all modules have taken a 45-degree angle forming a symmetrical circle, from this position can't be generate movement Because the angles can't change in the individual modules, also the configuration is unstable and it is necessary to lower the center of gravity.

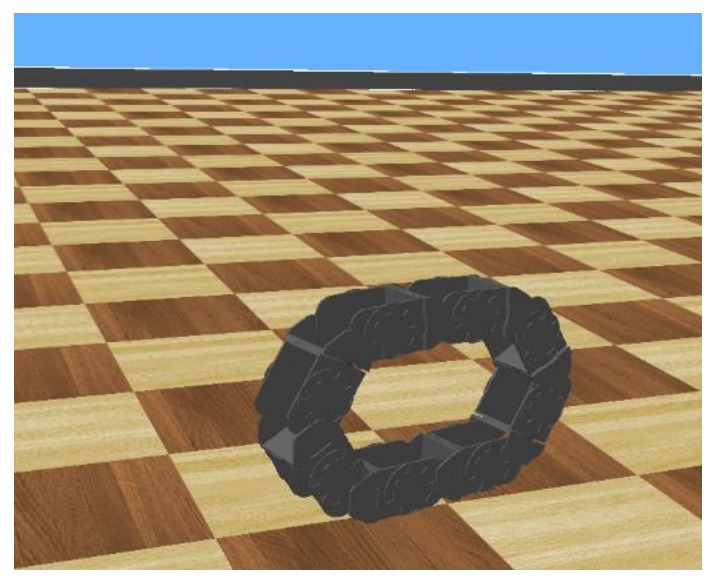

Figure 6: Simulated Movement Position

As solution to the problem of movement of joints and Lower the center of mass is created a position of movement whit polygonal shape as shown in the Figure 6, with this thing that is obtained is to be able to move the center of mass forward or back depending on the movement that want to obtain the displacement of the wheel is given keeping this defined geometry throughout the tour.

\section{MODULAR ROBOTIC SYSTEM MECABOT 4.0 IMPLEMENTATION}

The implementation of the system was made completely in the facilities of the Nueva Granada military University, where began by printing on ABS plastic of the required parts for the construction of four modules to form architecture. Once they are printed parts, put bolts and electronic elements in their place. It is highlighting that for this version a distribution was made by semi-modules, in a semi - module are located the elements of processing and control, in the other semi - module are food items such as batteries, voltage regulators and the respective driver. The Figure 7 . Shows the final result of the implementation of the module. 


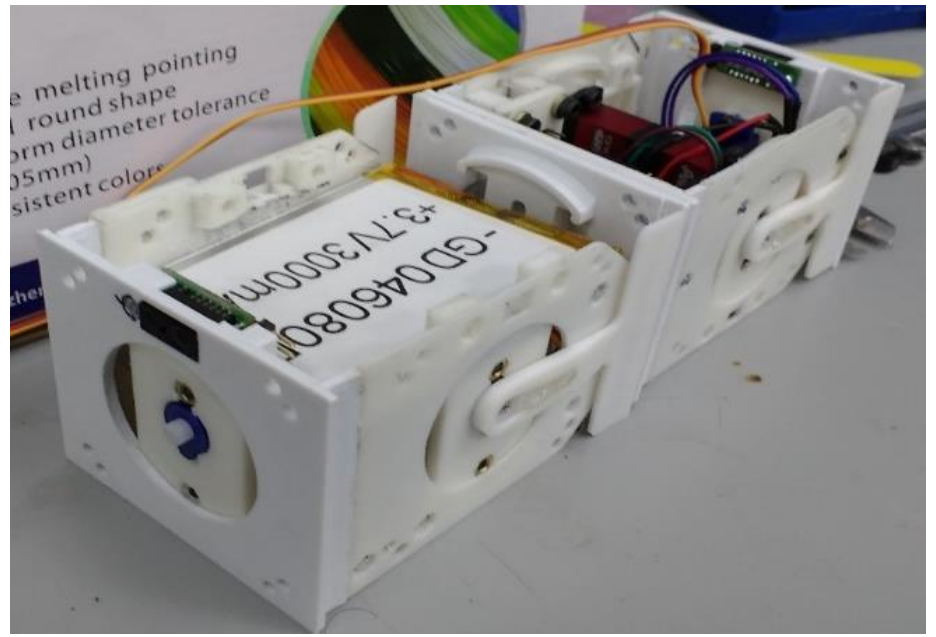

Figure 7: Result of the Implementation of Each Module

\section{CONSTRUCTION OF ARCHITECTURE TYPE WHEEL}

Once built the four necessary modules began to program each one according to his identification as everyone has a different function in each of step to run, followed by this power of the modules is proven to form the proposed geometry as shown in Figure 8.

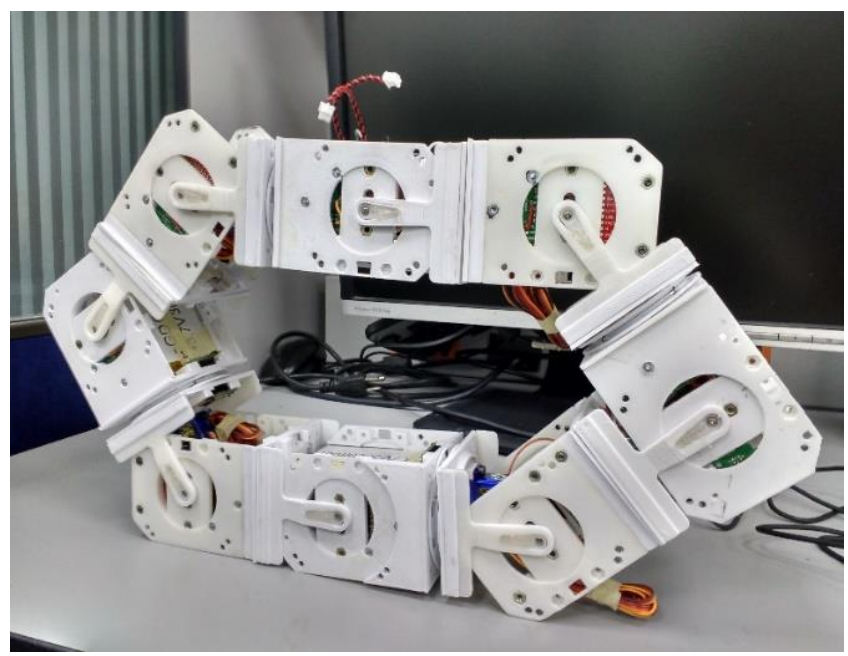

Figure 8: Modular Robotic System Powered by Batteries

At this point are presented some difficulties in the generation of movement since the actuators require more current that voltage regulators provide, for this reason sometimes modules are reset and the uniformity of the geometria is lost this problem is solve Connecting the system directly to the voltage source, voltage supply system in order to obtain the greater torque of actuators, in addition to this the current problem is mitigated because the voltage source provide up to $5 \mathrm{~A}$. The Figure 9 illustrates the geometry directly fed from the supply voltage. 


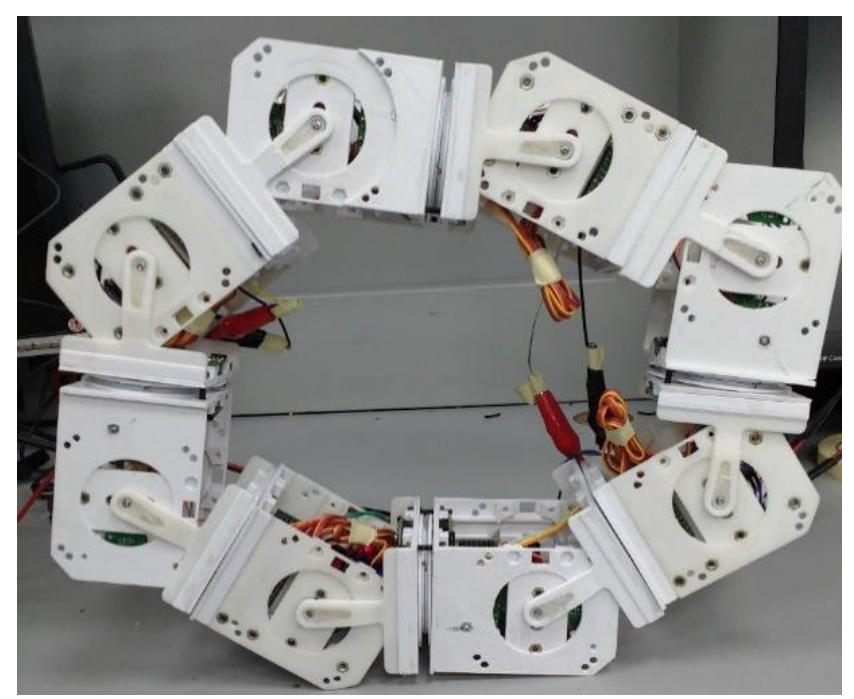

\section{Figure 9: Modular Robotic System Powered by Supply Voltage}

Besides this as support to the system decides to remove the batteries, this helps to reduce the weight of the modules and to retain geometry, the magnetic coupling system does not keep the modules together during the movement, so the union was reinforced with bolts.

\section{TESTING AND ANALYSIS OF MOVEMENT}

Once solved the problems mechanics becomes one with a measuring surface with $5 \mathrm{~cm}$ grid as shown in Figure 10 , this surface data was taken as total distance traveled, and distance travelled by step. They were also measured travel times.

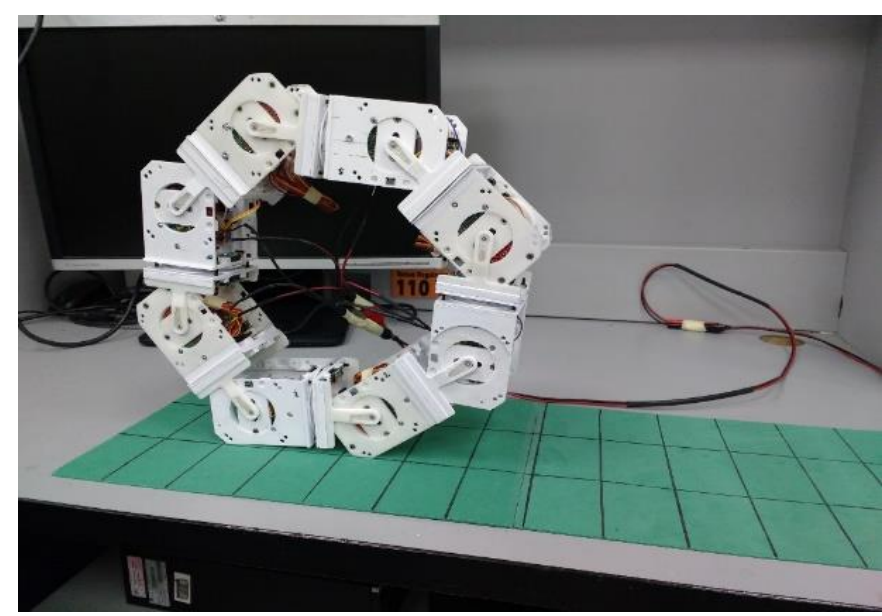

Figure 10: Structure on Grid Parameters Measurement

The measurement of these parameters seeks to obtain the rate as an indicator of system performance, among the tests that were done is various acceleration of the actuators and the frequency of steps, of the combination of these two parameters depends on the displacement of greater speed and fluidity that can achieve the wheel, in the figure 11 it can be seen the results of several accelerations of the actuators with constant step frequency. 


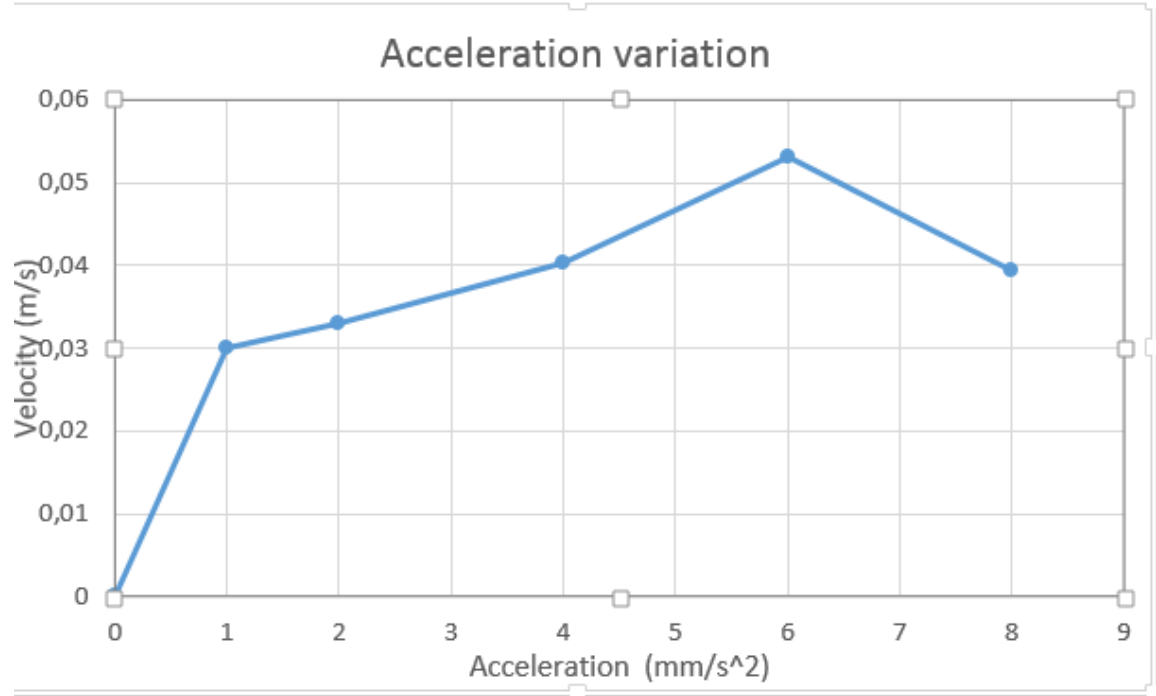

Figure 11: Results of Acceleration Variation.

In the previous figure can be seen that the higher speed was obtained with acceleration $6\left(\mathrm{~mm} / \mathrm{s}^{\wedge} 2\right)$, but the disadvantage is that movements are a bit broken off and slow, in addition to this you can see it with acceleration zero didnot have time so that engines reach perform their functions so there was no movement.

\section{Acceleration variation}

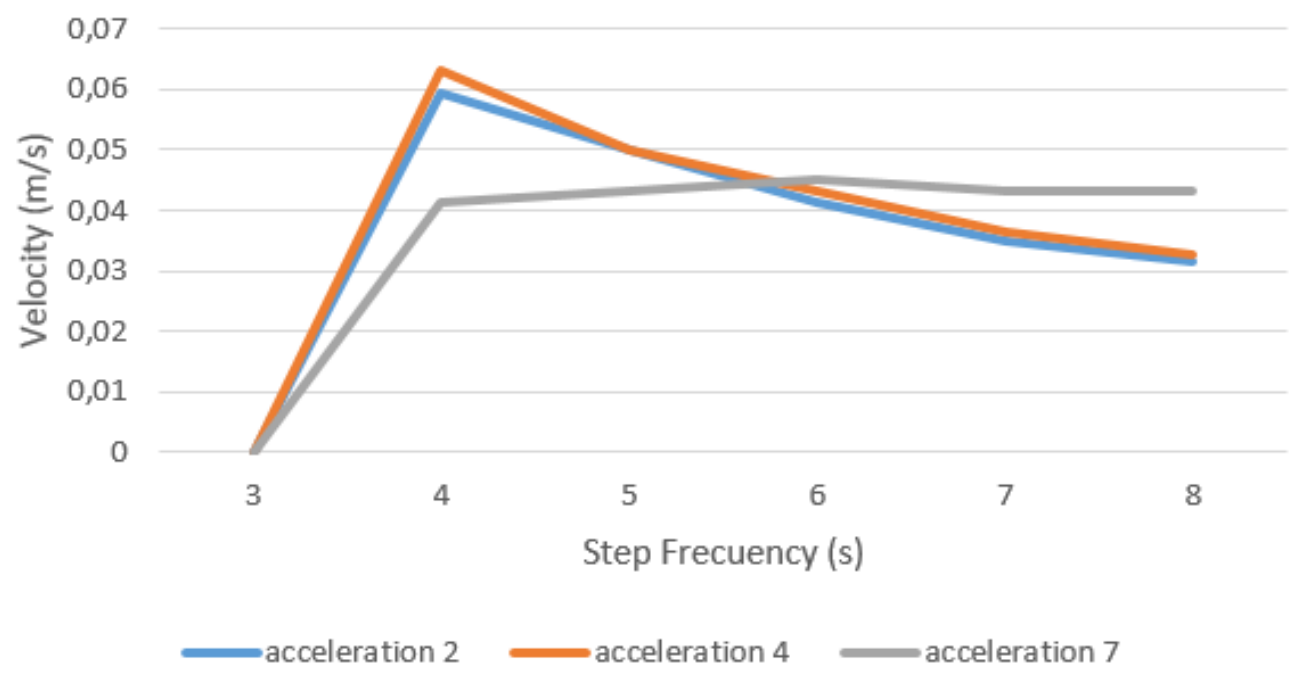

Figure 12: Results from variation of Accelerations and Step Frequency

In Figure 12. evidenced speed attained by combining the frequency with the acceleration of the actuators, here you can see that the highest speed achieved this with acceleration of $4\left(\mathrm{~mm} / \mathrm{s}^{\wedge} 2\right)$ and frequency of $4 \mathrm{~Hz}$, this movement was smooth and fluid, by increasing the acceleration of the actuators the geometry of the wheel was deformed, and by increasing the frequency of step the movement was slower.

\section{CONCLUSIONS}

It was compared and analyzed positive and negative aspects of acceleration and step frequency changes for wheel-type configuration from speed as a performance indicator for the modular robotic system Mecabot 4.0. 
It was verified the practicality of the frames programming of movements, thus allows a clear and more organized follow-up of the execution of sequences, as well as organize orders reception and sent, since each movement is a function and are called strictly when it is necessary in this form is better controlled the entire system.

By the results obtained in the implementation found approximation of the Webots Simulator to possess tools that allow finding physical properties of the modular robotic system and its environment allowing to obtain the values of speed and functionality.

It is not possible to ensure perfect symmetry of the architecture during the displacement due to the power of the actuators of the joints and total weight of the modular robotic system Mecabot 4.0.

\section{ACKNOWLEDGEMENTS}

The authors and DAVINCI Research Group would like to thank the Research Vice Deanery of Universidad Militar Nueva Granada for funding the INV-ING-2376 research Project, titled "Development of technique for architectural selection and automatic assembly of agents using modular robotics, based on work environment detection"from which this work is derived.

\section{REFERENCES}

1. Alonso-Puig, A. (2006). Application of Waves displacement algorithms for the generation of gaits in an all terrain hexapod. In Climbing and Walking Robots (pp. 343-348). Springer, Berlin, Heidelberg.

2. Arredondo, R. (2006). Design and simulation of locomotion of self-organising modular robots for adaptive furniture (Doctoral dissertation, Master's thesis, Swiss Federal Institute of Technology Lausanne, Biologically Inspired Robotics Group).

3. Bares, J. E., \& Wettergreen, D. S. (1999). Dante II: Technical description, results, and lessons learned. The International Journal of Robotics Research, 18(7), (pp. 621-649).

4. Borenstein, J., Hansen, M., \& Nguyen, H. (2006, February). The OmniTread OT-4 serpentine robot for emergencies and hazardous environments. In International Joint Topical Meeting: Sharing Solutions for Emergencies and Hazardous Environments (pp. 12-15).

5. Bourquin, Y., Ijspeert, A. J., \& Harvey, I. (2004). Self-organization of locomotion in modular robots. Unpublished Diploma Thesis.

6. Lyder, A., Garcia, R. F. M., \& Stoy, K. (2008, September). Mechanical design of odin, an extendable heterogeneous deformable modular robot. In 2008 IEEE/RSJ International Conference on Intelligent Robots and Systems (pp. 883-888).

7. Lyder, A., Garcia, R. F. M., \& Stoy, K. (2010). Genderless connection mechanism for modular robots introducing torque transmission between modules. In Proceedings of the ICRA Workshop on Modular Robots, State of the Art (pp. 77-81).

8. Dietsch, J., Moeckel, R., Jaquier, C., Drapel, K., Dittrich, E., Upegui, A., \& Ijspeert, A. J. (2006). Exploring adaptive locomotion with YaMoR, a novel autonomous modular robot with Bluetooth interface. Industrial Robot: An International Journal.

9. Mockel, R., Sprowitz, A., Maye, J., \& Ijspeert, A. J. (2007). An easy to use bluetooth scatternet protocol for fast data exchange in wireless sensor networks and autonomous robots. In 2007 IEEE/RSJ International Conference on Intelligent Robots and Systems (pp. 2801-2806).

10. Park, M., Chitta, S., Teichman, A., \& Yim, M. (2008). Automatic configuration recognition methods in modular robots. The International Journal of Robotics Research, 27(3-4), 403-421. 
11. Ryland, G. G., \& Cheng, H. H. (2010, May). Design of iMobot, an intelligent reconfigurable mobile robot with novel locomotion. In 2010 IEEE International Conference on Robotics and Automation (pp. 60-65).

12. IEEE. (2005) IEEE/ASME Transactions on Mechatronics, VOL. 10, $N^{\circ} .3,2005$.

\section{AUTHORS PROFILE}

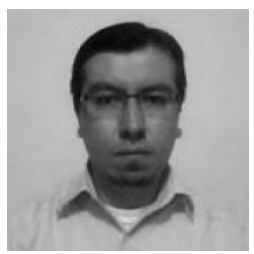

Ricardo A. Castillo was born in Colombia. He received his B.Eng. in Mechatronics Engineering in Universidad Militar Nueva Granada in 2004, and his M.Sc. and Ph.D. in Mechanical Engineering in the University of Campinas - UNICAMP, Brazil in 2010 and 2015 respectively for his work on coordination and indirect communication strategies for mechatronic systems. Since 2005 he is a full-time Professor and Researcher in the Department of Mechatronics Engineering at Universidad Militar Nueva Granada. His current research projects deal with collaborative modular robotics and mobile autonomous robotics.

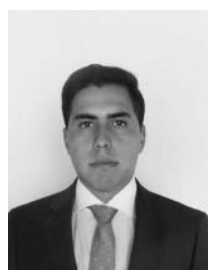

Wilhelm A. Miño was born on March 21th in Bogota, Colombia. He received his B.Eng. in Mechatronics Engineering in Universidad Militar Nueva Granada in 2017, he then linked to the university as a research assistant in 2018, working as the main topics robotics mobile, modular robotics, swarm and artificial intelligence.

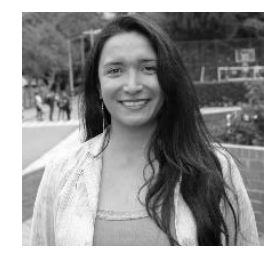

Nancy E. Olarte was born on March 22th in Bogotá, Colombia. She received his B.Eng. in Telecommunications Engineering in Universidad Militar Nueva Granada in 2009, she then linked to the university as a research assistant in 2011, working as the main topics telemedicine and virtual communication systems, and his M.Sc. in Information Technologies Applied to Education in Universidad Pedagógica Nacional - UPN, in 2016 respectively. Since 2014 she is a full-time Professor and Researcher in the Department of Electronics and Communications Technology at Universidad Militar Nueva Granada. 
